\title{
RELATO DE APLICAÇÃO DE PJBL NA ÁREA DE SISTEMAS ELÉTRICOS DE POTÊNCIA À ACADÊMICOS DE GRADUAÇÃO
}

\author{
Report of Application of PjBL in the Area of Electrical Power Systems with \\ Undergraduate Students
}

Carlos Eduardo Pupin ${ }^{1}$

Tania Mara Zancanaro Pieczkowski ${ }^{2}$

\begin{abstract}
Resumo: As discussões acerca dos processos de ensino e aprendizagem têm ganhado evidência na educação superior, com o objetivo de reduzir a evasão escolar e estimular o aprendizado. $\mathrm{O}$ presente artigo tem como objetivo relatar a experiência da aplicação de uma aprendizagem baseada em projeto, do inglês, project based learning ( $\mathrm{PjBL})$. $\mathrm{O}$ método ativo foi aplicado à uma turma das fases finais do curso de graduação em Engenharia Elétrica. No decorrer do trabalho são comentadas as etapas da aplicação do PjBL, os métodos de ensino utilizados, a contextualização do caso, os desafios enfrentados pelo docente, a resistência por parte dos acadêmicos, projeto escolhido e suas especificações, o método avaliativo aplicado e para concluir, os relatos do docente, referente às habilidades e competência adquirida pelos acadêmicos ao final do processo. A aplicação da metodologia ativa do tipo PjBL exige um grande desdobramento por parte do docente e dos acadêmicos, ampliando o tempo de trabalho fora de sala de aula, mas apresenta bons resultados no processo de aprendizagem. Ao final da componente curricular, na perspectiva do docente, ao comparar com modelo expositivo, há indícios que apoiam a melhora do aprendizado teórico, maior envolvimento e participação dos acadêmicos, que contribui com a formação de egressos melhor adaptados aos desafios profissionais.
\end{abstract}

Palavras-chave: Aprendizado baseado em projetos. Aplicação de metodologia ativa no ensino. Relato de experiência.

\begin{abstract}
Discussions about teaching and learning processes have gained evidence in higher education with the objective of reducing school dropouts and encouraging learning. This article aims to report the experience of applying project-based learning $(\mathrm{PjBL})$. The active method was applied to a group of the final phases of the Electrical Engineering undergraduate course. During the work, the stages of the application of PjBL are discussed; the teaching methods used; the contextualization of the case; the challenges faced by the teacher; the academics resistance; the chosen project and its specifications; the applied evaluation method and for conclude; the teacher reports, referring to the skills and competence acquired by the students at the end of the process. The application of PjBL active methodology requires a great deal of development on the part of teacher and academics, increasing work time outside the classroom, but it presents good results in the learning process. At the end of the curricular component, from the teacher's perspective, when compared to the expository model, there are signs that support
\end{abstract}

\footnotetext{
${ }^{1}$ Doutor em Engenharia Elétrica. Professor Titular da Universidade Comunitária da Região de Chapecó Unochapecó, Chapecó/SC. carlos.pupin@ unochapeco.edu.br - https://orcid.org/0000-0002-2342-491X

2 Doutora em Educação. Professora Titular na graduação e pós graduação da Universidade Comunitária da Região de Chapecó - Unochapecó, Chapecó/SC. taniazp@ unochapeco.edu.br - https://orcid.org/0000-0002-5257-7747
} 
the improvement of theoretical learning, greater involvement and participation of academics, which contributes to the formation of graduates better adapted to professional challenges.

Keywords: Project based learning. Active methodology application in education. Experience report.

\section{Introdução}

A aprendizagem baseada em problemas (problem-based learning - PBL) e a aprendizagem baseada em projetos (project-based learning - PjBL) são modelos contemporâneos de ensino, muitas vezes, denominados apenas como PBL. Esses modelos são baseados em metodologias ativas de aprendizagem, sendo as atividades projetadas com foco nos resultados, devendo ao longo do seu desenvolvimento, promover a reflexão nos alunos e fazer com que eles pensem sobre o que estão aprendendo (PRINCE, 2004).

Esses modelos apresentam ampla aceitação nas Universidades de todo o mundo e têm recebido ênfase nos últimos anos, sendo empregados em diversas áreas do conhecimento, incluindo Medicina, Administração, Ciências e Engenharias (KOLMOS; GRAAFF, 2014). No caso específico das Engenharias, é vasto o campo de problemas e/ou projetos que podem ser utilizados. No entanto, uma questão fundamental a ser investigada é se esses novos modelos são tão eficientes quanto ou mais para aprendizagem do que os modelos tradicionais ${ }^{3}$ de ensino.

Sabe-se que a disponibilidade suficiente de engenheiros é estratégica para que a economia de um país tenha uma posição competitiva (VILLAS-BOAS; GIOVANNINI, 2014). Na década de 1960 houve uma grande necessidade de profissionais qualificados para suprir a demanda do setor industrial, acarretando na expansão do ensino superior. Até 1950, existiam apenas 16 instituições de educação superior no Brasil que ofereciam 62 cursos de Engenharia (OLIVEIRA et al., 2013). A Sinopse Estatística da Educação Superior (Instituto Nacional de Estudos e Pesquisas Educacionais Anísio Teixeira - INEP) aponta que, atualmente, o país apresenta 1.238 instituições de educação superior que oferecem cursos de Engenharia, Produção e Construção, das quais 457 referem-se, especificamente, ao curso de Engenharia Elétrica. Por esse banco de dados, em 2019, existiam 98.819 alunos matriculados no curso, sendo 11.784 concluintes (menos de 12\%). O número de matrículas trancadas era de 14.859 e 25.765 matrículas desvinculadas. Em ambos os casos, as Universidades privadas eram as mais afetadas, com cerca de 82\% de desistência dos discentes (INEP, 2019). Diante desses números, percebe-se uma taxa elevada de evasão do curso que, portanto, resulta em uma ineficiência do sistema educacional (OLIVEIRA et al., 2013; DAHMS, 2014; FURTADO; NASCIMENTO; SILVA, 2018).

Além da evasão, o método de aula tradicional dentro do ensino de Engenharia é dedutivo (YADAV et al., 2011). Inicia-se com a apresentação de teorias e progride em direção a aplicação dessas teorias, sem uma discussão de por que os modelos matemáticos estão sendo desenvolvidos e quais problemas práticos poderão resolver (PRINCE; FELDER, 2006). Em seu livro, Dewey, ainda em 1938, destaca que nos métodos tradicionais o professor é o centro de transmissão do conhecimento, cabendo aos alunos receber, assimilar e memorizar o conteúdo, tornando o ambiente de aprendizagem abstrato, monótono e deixando os alunos com uma sensação de tédio e falta de motivação (DEWEY, 1938). Cabe destacar ainda, que nos modelos

\footnotetext{
3 Como tradicional entenda-se o modelo de aprendizagem expositivo, em que o acadêmico não atua ativamente no processo de aprendizagem.
} 
tradicionais, muitas vezes, há apenas uma resposta correta para cada problema, o que pode limitar o aprofundamento do conhecimento e a criatividade (CIFRIAN et al., 2020). Brown, Collins e Duguid (1989) destacaram que, a menos que o conhecimento seja desenvolvido no contexto em que será aplicado, os alunos poderão compreender conceitos, algoritmos e procedimentos, mas o conhecimento permanece inerte e, dificilmente, os alunos conseguirão empregá-lo.

Para auxiliar o desenvolvimento de competências e habilidades nos acadêmicos dos cursos de graduação e pós-graduação e contornar os desafios de aprendizagem, houve muitas mudanças nos sistemas de ensino nos últimos 40 a 50 anos. Os modelos de aprendizagem com novas abordagens pedagógicas se originaram a partir de reformas nas Universidades entre os anos de 1965 e 1975, em resposta às necessidades da sociedade. As novas Universidades foram importantes para otimizar três aspectos: $i$ ) eram necessários novos conhecimentos e as habilidades desenvolvidas deveriam ser dirigidas para o mercado de trabalho; $i$ ) os programas de estudo eram muito fragmentados e sem relação com mundo exterior; iii) havia a necessidade da universidade de ser mais democrática e da atuação estudantil ser mais enfática (KOLMOS; GRAAFF, 2014).

Os (não tão) novos modelos de aprendizagem foram aplicados, primeiramente, na Suécia, Austrália, Holanda, Dinamarca, Alemanha, Cingapura e em muitos outros países. A primeira instituição de ensino superior a aplicar modelos baseados em PBL foi a Universidade de McMaster, no Canadá, ainda na década de 70 (NEUFELD; BARROWS, 1974), que tinha como princípio: os problemas constituem o foco e estímulo para aprendizagem; os problemas são veículo para o desenvolvimento de habilidades para solucioná-los; as novas informações devem ser obtidas por meio da aprendizagem autodirigida; o método deve ser centrado no aluno; para prática, são formados pequenos grupos de alunos e os professores atuam como guias/facilitadores (BARROWS, 1996). No modelo da Universidade McMaster, a grade curricular foi dividida em blocos temáticos, sendo cada ano dividido em uma série de blocos, de aproximadamente seis semanas. Cada bloco apresenta uma série de casos que os alunos devem analisar, sendo que, cada caso, refere-se a um problema que irá desencadear o processo de aprendizagem pelo aluno e não precisa ser, necessariamente, resolvido (KOLMOS; GRAAFF, 2014).

De fato, a Engenharia exige que seus profissionais enfrentem incertezas e resolvam problemas complexos. Além disso, é preciso que sejam membros eficazes de equipes e tenham habilidade de comunicação (YADAV et al., 2011). No PBL, os problemas constituem o ponto de partida para a aprendizagem autodirigida em equipes. Desse modo, os alunos analisam e discutem os casos em grupos, definindo metas de aprendizagem que serão estudadas individualmente. Os resultados dos estudos individuais são relatados e a discussão ocorre novamente em grupo, para dar continuidade e para que as novas atividades sejam distribuídas entre os integrantes da equipe (KOLMOS; GRAAFF, 2014).

Partilhando essa característica, no modelo PjBL, introduzido nas Universidades de Aalborg e Roskilde (Dinamarca), os alunos trabalham o problema em equipes, mas como componente adicional, há necessidade de elaboração de um relatório concluído de modo colaborativo pela equipe. $\mathrm{O} \mathrm{PjBL}$ inicia-se com um problema que será trabalhado pelo grupo (cinco a oito alunos). Normalmente, os próprios alunos definem o problema dentro de um quadro temático. Nesse caso, a realização de uma ou mais atividades resultarão em um produto final como, por exemplo, um modelo, um dispositivo ou uma simulação computacional. No processo de aprendizagem, os alunos lidam com conteúdos interdisciplinares, tomam decisões e atuam de modo individual e em equipe. O relatório é apresentado em forma escrita e/ou oral, em que é exposto o procedimento e apresentado os resultados. A avaliação do aluno é pautada 
no desempenho durante a realização do projeto e na entrega do relatório. Um aspecto interessante do PjBL é que ele oferece ao aluno competências para o desenvolvimento de um projeto, desde como iniciar até a finalização, algo que é pretendido pelas empresas (KOLMOS; GRAAFF, 2014).

Dentro da implementação dos modelos de PjBL, os projetos podem ser classificados em construtivo, explicativo ou investigativo e variar de curta (uma a duas semanas) a longa duração (um a dois semestres). No primeiro caso, os projetos são restritos ao ambiente de sala de aula e baseados em objetos de conhecimento específicos. No segundo, pode envolver soluções complexas, que demandam temas transversais e resoluções interdisciplinares (MORAN, 2018).

As diferenças básicas entre esses novos modelos de aprendizagem residem no fato de que, na aprendizagem baseada em $\mathrm{PjBL}$, os alunos recebem o conhecimento básico necessário por meio da instrução formal e o foco está fundamentado no produto final, enquanto no PBL, os alunos trabalham em problemas enquanto adquirem a base do conhecimento necessário para completar a atividade e, portanto, o foco está no processo de aprendizagem ao invés do produto final (YADAV et al., 2011). Uma questão fundamental exige que, no PjBL tanto alunos como professores, monitorem, reflitam, avaliem e atualizem suas práticas continuamente, pois o processo de aprendizagem não pode ser totalmente pré-determinado.

De modo geral, ambos modelos possuem como ponto de partida um problema da vida real a ser solucionado, exigindo conhecimentos complexos e tomada de decisão. Isso consiste em uma forte motivação que é oferecida aos alunos, fator fundamental para o engajamento no projeto, no caso do $\mathrm{PjBL}$.

As metodologias ativas são estratégias de aprendizagem que buscam estimular o auto engajamento do acadêmico, que ao vislumbrar um evento científico, se entusiasma em aprimorar (autorregulação) seus conhecimentos, confrontando-o ao seu conhecimento prévio (metacognição) (SANTOS 2015). Para Berbel (2011), a implementação de metodologias ativas motiva a autonomia do aluno por buscar soluções às situações problemas vivenciados por ele, desenvolvendo o protagonismo, culminando com soluções criativas.

Segundo Bordenave e Pereira (1982), um bom projeto deve passar por quatro diferentes fases, a de:

a) despertar curiosidade, intenção de solução, para isso deve ser uma situação real e próxima ao acadêmico;

b) estimular a investigação/pesquisa para obtenção de novos conhecimentos;

c) permitir a execução, para que o acadêmico aprenda fazendo; e

d) apreciação, com avaliação crítica sob o trabalho final.

Os aspectos positivos e reconhecidos da metodologia $\mathrm{PjBL}$ centram-se no fato de que os alunos desenvolvem, além de competências profissionais da Engenharia, habilidades metodológicas como gestão de projetos, trabalho em equipe, negociação, comunicação e resolução de problemas (DAHMS, 2014). Outros benefícios incluem o incentivo aos alunos a desenvolverem abordagens equilibradas, porém diversificadas para resolver problemas do mundo real. Assim, eles conseguem entender a relevância das informações a serem estudadas e são ajudados a aprender a informação da mesma forma em que ela será aplicada na prática (CIFRIAN et al., 2020).

Apesar disso, o processo de mudança educacional é complexo e deve incluir o desenvolvimento de pelo menos três elementos: currículo, equipe e instituição. O primeiro item consiste no desenvolvimento do novo currículo, que deve incluir os resultados de 
aprendizagem, avaliações e atividades de aprendizagem e ensino; o segundo enfatiza a preparação dos docentes, que altera a abordagem tradicional focada no professor para abordagem centrada no aluno. Por fim, o desenvolvimento institucional inclui alterações no layout e no uso da infraestrutura física, formação de sistemas de apoio ao aluno e acesso aos recursos de aprendizagem (DAHMS, 2014).

A metodologia $\mathrm{PjBL}$ tem demonstrado melhorias nos resultados de aprendizagem, em comparação com as práticas de ensino tradicionais, baseada em aulas teóricas e exercícios práticos, em que os alunos são apenas receptores passivos de informação. Relatos positivos foram obtidos por Cifrian et al. (2020) que destacaram a experiência adquirida pelos alunos de Engenharia Química, o aprimoramento de suas estratégias de aprendizagem e maior motivação, como obstáculos relataram necessidade de mudanças organizacionais e a formação continuada dos professores, com incentivo para inovação. Cecílio e Tedesco (2019) sugeriram que a aplicação dos novos modelos de aprendizagem deve ser bem planejada e a interação com os alunos deve ser constante. A mediação foi um ponto importante, uma vez que, de acordo com os autores, permitiu envolver os alunos em um equilíbrio de transferência de conhecimento. Furtado, Nascimento e Silva (2018) verificaram que as atividades realizadas de forma ativa pelos alunos estimularam as habilidades de comunicação e o trabalho em equipe.

Yadav et al. (2011) relataram que os ganhos de aprendizagem dos alunos na disciplina de Eletricidade Básica foram o dobro em relação aos modelos tradicionais de ensino. Neste estudo foram realizados pré e pós-testes, e a diferença entre a nota do acadêmico no pós-teste em relação ao pré-teste é definida como ganho de aprendizagem. Os alunos que tiveram aula pelo método PBL tiveram ganhos de aprendizagem maiores que o dobro do expositivo, a coleta de dados e o método de avaliação seguem conforme (GAY; MILLS; AIRASIAN, 2006), e as a análise estatística e de escalas constam em (COHEN, 1988).

Assim, verifica-se que é encorajadora a aplicação de novas metodologias de ensino com foco no aluno. Diante desse cenário, o objetivo desse estudo é relatar, do ponto de vista do docente, a experiência de aplicar a metodologia de ensino e aprendizagem baseada em projetos, em uma turma da $8^{a}$ fase do curso de Engenharia Elétrica.

O presente material é fruto do curso de especialização em Aprendizagem Ativa e Inovação Acadêmica fornecido como forma de formação continuada ofertada pela Universidade Comunitária da Região de Chapecó (UNOCHAPECÓ), que ofertou o curso para professores e gestores da instituição, objetivando a reestruturação dos projetos pedagógicos dos cursos de graduação, incentivando a aplicação de práticas docentes que envolvam metodologias ativas, ou formas inovadoras de conduzir a aula universitária.

\section{Contextualização}

Os relatos presentes neste trabalho discorrem sobre a aplicação da metodologia de ensino PjBL, no ponto de vista do docente, praticado no segundo semestre do ano de 2020, na componente curricular Proteção de Sistemas Elétricos de Potência, da $8^{a}$ fase do curso de graduação de Engenharia elétrica da Universidade Comunitária da Região de Chapecó (UNOCHAPECÓ).

A disciplina deve fornecer ao acadêmico a habilidade de avaliar, dimensionar e projetar um sistema de proteção para redes elétricas de grande potência, comprometido com sua operação segura e confiável. Faz parte das componentes específicas, nas fases finais do curso de graduação. Concentra grande carga de conhecimento técnico prévio, por exemplo, circuitos elétricos, máquinas elétricas e sistemas elétricos de potência. 
A turma apresentava a quantidade ideal de acadêmicos para a aplicação do PjBL, no total de 10, de diferentes faixas etárias (de 20 até 40 anos). Foi o primeiro momento que estes acadêmicos vivenciaram a metodologia $\mathrm{PjBL}$, até então, era predominante o método expositivo.

Vale ressaltar que o componente curricular ocorreu por videoconferência, com participação síncrona ${ }^{4}$ dos acadêmicos e professor, devido ao período de pandemia da Covid19. Tal fato dificultou o compartilhamento e a socialização entre os membros do grupo durante o horário de aula, o que também dificultou o docente de acompanhar os assuntos partilhados dentro dos grupos.

\section{Delimitações do PjBL}

A aplicação do PjBL deve iniciar pela escolha do projeto a ser desenvolvido. O docente deve previamente avaliar quais os resultados de aprendizagem que serão atingidos pelos acadêmicos ao desenvolver tal projeto, que deve:

a) ser envolvente, estimulando o acadêmico a pesquisar sobre o assunto, tornando prazeroso o seu desenvolvimento. A construção do trabalho deve fluir naturalmente, e não porque vale nota;

b) ser desafiador para a área de Engenharia, mas não demasiadamente complexo. A experiência profissional do docente é determinante, podendo por exemplo, se utilizar de casos reais;

c) revisar assuntos anteriormente trabalhados durante a graduação, de preferência, que a aplicação desse conhecimento gere dados iniciais necessários à sua elaboração, agregando significância ao assunto revisado;

d) explicitar os objetivos a serem atingidos, e evidenciar como será o processo avaliativo e demais rubricas advindas de sua realização.

O projeto deve ser disponibilizado com o máximo de informações e os objetivos esclarecidos com a turma, ainda nas primeiras aulas, para nortear os estudos que serão posteriormente desenvolvidos de forma autônoma pelos grupos.

Por se tratar de um conteúdo que envolve cálculos complexos, algumas aulas permanecem na metodologia expositiva, para compartilhar conhecimento básico e experiências em trabalhar com PBL. Embora caiba ao professor criar ambientes fecundos para a aprendizagem, a obtenção dos conhecimentos teóricos requer o protagonismo do acadêmico.

\section{Relato da aplicação do PjBL}

Na situação vivenciada pela turma de Proteção de Sistemas Elétricos de Potência de 2020/2, o projeto consistia na elaboração de um estudo de proteção para um sistema elétrico de alta tensão composto por trechos de geração, transmissão e carga, elaborado pelo docente com os típicos dados que seriam conhecidos em uma situação real de projeto. O projeto foi trabalhado durante todo o semestre letivo.

A turma foi dividida em duplas, apesar de na metodologia $\mathrm{PjBL}$ o compartilhamento de saberes ser melhor aplicado em grupos de 5 a 8 acadêmicos. Devido à inexperiência dos alunos em (com)partilhar o conhecimento, o docente priorizou a divisão em duplas. A dificuldade, antes mencionada, ficou evidente ao longo do desenvolvimento do projeto, pois na

\footnotetext{
${ }^{4}$ Por síncrona entenda a participação simultânea dos envolvidos em tempo real.
} 
maioria das duplas cada acadêmico preferiu por desenvolver individualmente o algoritmo de cálculo, para conferência dos resultados com o parceiro.

$\mathrm{Na}$ mesma lógica, dentre as cinco duplas, três realizaram os projetos pelo método de divisão de tarefas, ou seja, o projeto final entregue foi segmentado e cada acadêmico se responsabilizou por elaborar um trecho deste trabalho. Essa postura evidenciou a inexperiência dos acadêmicos em trabalhar em grupo. Possivelmente, o resultado seria diferente, se a metodologia PBL se iniciasse nas primeiras fases do curso de graduação, ou até mesmo no ensino médio.

Para Dewey (1938), "Um projeto prova ser bom se for suficientemente completo para exigir uma variedade de respostas de diferentes alunos e permitir a cada um trazer uma contribuição que lhe seja própria e característica". Mantendo esta ideia, um único sistema elétrico foi disponibilizado, para desenvolvimento do projeto, e cada grupo foi responsável por projetar e ajustar as proteções aplicadas em um trecho específico do sistema elétrico, compreendendo por exemplo, a proteção dos geradores, transformadores, barramentos, linhas de transmissão e cargas. Cada um destes equipamentos descritos possui tipos distintos de proteção, e obrigatoriamente deve existir a coordenação e seletividade entre os equipamentos deste trecho, mas essas duas exigências também devem ser garantidas para os demais grupos, obrigando a interação e compartilhamento de resultados entre todos os demais grupos. Só a observação destes fatores garante o correto dimensionamento do projeto.

O início do projeto se deu pelo cálculo da corrente de curto circuito, um dado fundamental para iniciar os cálculos de ajustes da proteção. Esse conteúdo foi trabalhado na disciplina do semestre anterior, e é um pré-requisito. Nesse momento, o grupo foi mobilizado para desenvolver os cálculos utilizando um recurso computacional, como Excel, Matlab, Mathcad etc.

Na sequência, foram dimensionados os demais equipamentos como transformadores de corrente e de potencial, os relés de proteção, disjuntores, seccionadoras, para-raios etc.

Por fim, com as informações obtidas anteriormente, foi realizado o estudo de proteção, definindo as proteções utilizadas e seus ajustes. Nesse momento, os acadêmicos avaliaram a atuação da proteção se baseando nos estudos teóricos anteriormente realizados, e o resultado não deveria ser compreendido como uma única resposta, e sim, perceber a existência de inúmeras respostas que podem ser consideradas corretas, dentro de limites máximos e mínimos, definidos pelos equipamentos protegidos do grupo e dos grupos vizinhos.

Nesse momento, os grupos interagiram, trocaram informações técnicas e também utilizaram da boa comunicação e argumentação para chegar ao consenso.

Devido à pandemia da Covid-19 e a realização dos encontros por videoconferência, a interação dos grupos foi dificultada e a aplicação do PjBL foi, em partes, afetada. Ainda assim, os resultados obtidos foram de significativo avanço no processo de ensino aprendizagem, pois essa interação não ocorria na metodologia expositiva.

Para atingir os resultados de aprendizagem esperados, o método avaliativo teve que ser completamente adaptado. A avaliação ocorreu predominantemente sob a apresentação de seminários, que ocorreram a cada duas semanas, e na correção do relatório final do projeto.

Importante destacar que todas as avaliações aplicadas foram do docente avaliando os resultados apresentados pelos acadêmicos, usando como base os objetivos definidos no plano de ensino. O processo avaliativo poderia ser melhorado aplicando a autoavaliação e avaliação por pares. 


\section{Resultados atingidos}

A aplicação do PjBL foi um grande desafio ao docente e aos acadêmicos, dada a inexperiência de ambos nesse tipo de método de ensino, principalmente, para o docente é necessário um grande planejamento prévio devendo planejar qual tipo de projeto será aplicado, quais os dados técnicos mínimos fornecidos, quais os resultados de aprendizagem esperados e como avaliar o aprendizado dos acadêmicos.

Inicialmente, alguns acadêmicos apresentaram revolta pelo novo método, houve desinteresse em iniciar o desenvolvimento do projeto. Assim que efetivamente começaram a realizar os cálculos, foram aos poucos se envolvendo com o processo e ao final o projeto acabou superando as exigências iniciais.

Ao longo do semestre, diversas adaptações foram realizadas, para contornar alguns problemas que vão surgindo no desenvolvimento do projeto. Quanto mais detalhado, com o máximo possível de informações técnicas fornecidas, mais os acadêmicos tiveram liberdade para trabalhar indo além dos objetivos iniciais, trazendo novos conteúdos, situações problemas, inovações tecnológicas, e aplicando a interdisciplinaridade.

Dos avanços notados do método PjBL pode-se citar:

a) aumento de comunicação interna e externamente aos grupos, compartilhando e necessitando de resultados obtidos pelos demais grupos;

b) utilização de diversos recursos tecnológicos para desenvolvimento do projeto;

c) melhor desenvoltura na apresentação de seminários;

d) desenvolvimento da proatividade individual (autorregulação).

Concluindo, há indícios que a metodologia PBL é válida e traz ao final, resultados de aprendizagem que normalmente não são trabalhados no método tradicional de aprendizagem, como por exemplo, as soft skills. Com relação ao objeto de conhecimento, é similar ao do método tradicional, pois permanece a cargo do docente e na forma com que orienta os acadêmicos. Por fim, o planejamento da disciplina requer constantes esforços do docente, e uma grande disposição à adaptação.

\section{Agradecimentos}

Os autores agradecem a Universidade Comunitária da Região de Chapecó (UNOCHAPECÓ) pelo apoio à formação continuada ofertada por meio do curso de especialização em Aprendizagem Ativa e Inovação Acadêmica oferecido gratuitamente ao autor e demais docentes da instituição.

\section{Referências}

BERBEL, Neusi. As metodologias ativas e a promoção da autonomia de estudantes.

Semina: Ciências Sociais e Humanas, Londrina, v.32, n.1, p. 25-40, 2011. Disponível em: https://edisciplinas.usp.br/mod/resource/view.php?id=2987706. Acesso em: 29 jul. 2021.

BARROWS, Howard S. Problem-based learning in medicine and beyond: A brief overview. In: WILKERSON, Luann \& GIJSELAERS, Wim H. (Eds.) Bringing problem-based 
learning to higher education: Theory and practice. New Directions for Teaching and Learning No. 68. San Francisco, CA: Jossey-Bass. 1996. p.3-12.

BORDENAVE, Juan; PEREIRA, Adair. Estratégias de ensino-aprendizagem.

4. ed. Petrópolis: Vozes, 1982.

BROWN, John Seely; COLLINS, Allan; DUGUID, Paul. Situated cognition and the culture of learning. Educational Researcher, New York, v.18, n.1, p. 32-42, 1989.

CECÍLIO, Waléria Adriana Gonçalez; TEDESCO, Daniel Guimarães. Aprendizagem baseada em projetos: relato de experiência na disciplina de geometria analítica. Revista Docência do Ensino Superior, Belo Horizonte, v.9, p.1-20, 2019. Disponível em: https://doi.org/10.35699/2237-5864.2019.2600. Acesso em: 22 mar. 2021.

COHEN, Jacob. Statistical Power Analysis for the Behavioral Sciences. Hillsdale, New Jersey. Lawrence Erlbaum Associates. 1988. xxi, 567 p. 2nd ed. Disponível em: https://doi.org/10.4324/9780203771587. Acesso em 28 jul. 2021.

CIFRIAN, Eva et al. Integration of different assessment approaches: application to a projectbased learning engineering course. Education for Chemical Engineers, Frankfurt, v.31, p. 62-75, 2020. Disponível em: https://doi.org/10.1016/j.ece.2020.04.006. Acesso em: 15 mar. 2021.

DEWEY, John. Experience and education. New York: Collier and Kappa Delta Pi. 1938.

DAHMS, Mona-Lisa. Problem Based Learning in Engineering Education. In: Villas Boas, Valquíria \& Giovannini, Odilon (Eds), Attracting Young People to Engineering: ALE 2014. Brasília, DF. Associação Brasileira de Educação em Engenharia (Abenge). 2014.

FURTADO, Anderson Elias; NASCIMENTO, Denise Ferreira Laurito; SILVA, José Wilson de Jesus. Problem-Based Learning (PBL) applied simultaneously to 3rd and 7th-semester engineering students as a motivational tool. Revista Práxis, Volta Redonda, v.10, n.19, p.3343, 2018.

GAY, Lorie; MILLS, Geoffrey; AIRASIAN, Peter. Educational research: Competencies for analysis and applications. Upper Saddle River, NJ: Merrill Prentice Hall, 8th ed. 2006.

INEP, Instituto Nacional de Estudos e Pesquisas Educacionais Anísio Teixeira. Sinopse Estatística da Educação Superior - 2019. Disponível em: <http://inep.gov.br/sinopsesestatisticas-da-educacao-superior>. Acesso em: 15 mar. 2021.

KOLMOS, Anette; GRAAFF, Erik de; Problem-Based and Project-Based Learning in Engineering Education: Merging Models. In: JOHRI, Aditya; OLDS, Barbara M. (Orgs). Cambridge Handbook of Engineering Education Research. Cambridge, Cambridge University Press, 2014, p. 141-161. Disponível em: < DOI:10.1017/CBO9781139013451.012>. Acesso em: 15 mar. 2021.

MORAN, José. Metodologias ativas para uma aprendizagem mais profunda. In: MORAN, José; BACICH, Lilian (Org.). Metodologias ativas para uma educação inovadora: uma 
abordagem teórico-prática. Porto Alegre: Penso, 2018. Disponível em:

<http://www2.eca.usp.br/moran/wp-content/uploads/2013/12/metodologias.pdf >. Acesso em: 15 mar. 2021.

NEUFELD, Victor R.; BARROWS, Howard S. The "McMaster Philosophy": an approach to medical education. Journal of Medical Education, Teerã, v.49, n.11, p.1040-1050, 1974.

PRINCE, Michael J. Does active learning work? A review of the research. Journal of Engineering Education, Washington, v.93, n.3, p.223-232, 2004.

PRINCE, Michael J.; FELDER, Richard M. Inductive Teaching and Learning Methods: Definitions, Comparisons, and Research Bases. Journal of Engineering Education, Washington, v.95, n.2, p.123-138, 2006.

SANTOS, C. A. M. dos. O uso de Metodologias Ativas de aprendizagem a partir de uma perspectiva interdisciplinar. In: Congresso Nacional de Educação, 12, 2015, Curitiba PR. Curitiba PR, 2015, p. 27202-27212. Disponível em < http:// educere.bruc.com.br/arquivo/pdf2015/20543_10759.pdf>. Acesso em 21/10/2021.

OLIVEIRA, Vanderlí Fava de et al. Um Estudo Sobre a Expansão Da Formação em Engenharia no Brasil. Revista de Ensino de Engenharia, Brasília, v.32, n.3, p.1-20, 2013.

VILLAS BOAS, Valquíria; GIOVANNINI, Odilon. Attracting Young People to Engineering: ALE 2014. Associação Brasileira de Educação em Engenharia (Abenge), Brasília, 2014, 368 p.

YADAV, Aman et al. Problem-based Learning: Influence on Students' Learning in an Electrical Engineering Course. Journal of Engineering Education, Washington, v. 100, n.2, p.253-280, 2011.

Recebido em julho de 2021.

Aprovado em outubro de 2021. 